\title{
A NEW DESIGN MODEL FOR ADHESIVE JOINTS USED TO BOND FRP LAMINATES TO STEEL BEAMS
}

\author{
REZA HAGHANI \\ Assistant Professor \\ Chalmers University of Technology \\ Civil and Environmental Engineering, Sven Hultins gata 8, 412 96, Gothenburg, Sweden \\ Reza.haghani@chalmers.se* \\ MOHAMMAD AL-EMRANI \\ Associate Professor \\ Chalmers University of Technology \\ Civil and Environmental Engineering, Sven Hultins gata 8, 412 96, Gothenburg, Sweden \\ Mohammad.al-emrani@chalmers.se
}

\begin{abstract}
The strengthening and repair of existing structures using bonded carbon fiber reinforced polymer, CFRP, laminates has attracted a great deal of attention in the past two decades. Investigations clearly indicate the great potential of this method for restoring the capacity of corroded steel beams and improving their fatigue life. One important issue regarding the use of this technique in strengthening steel structures is the design of adhesive joints used to bond FRP laminates to steel substrates. Very limited research work has been conducted in this area and, at the present time, there is a lack of suitable design models for FRP-strengthened steel members. This paper is mainly concerned with a proposal for and verification of a new design model for adhesive joints used to bond FRP laminates to steel beams for strengthening and repair purposes. Quasi-static tests were performed on steel plate and full-scale beam specimens bonded with CFRP laminates to evaluate the new design model proposed in this study. The failure, in all specimens, took place at the steel-adhesive interface. The new design model presented in this paper was found to be accurate in terms of predicting the ultimate load and failure mode of the joints.
\end{abstract}

Keywords: Adhesive joint, Design , Flexural, FRP, Laminate, Steel

\section{Introduction}

The use of fiber reinforced polymer, FRP, laminates for the strengthening and repair of structures offers many advantages in comparison to traditional strengthening methods [1]. In recent years, there has been a trend towards using this technique to strengthen and repair structural steel members [2]. Although the effectiveness of the method for the strengthening and repair of steel members has been demonstrated in a number of studies, it can be seen that the technique is not yet as widespread as it is for concrete structures. A study of the literature indicates that, in order further to develop the applications of this strengthening method in the field of steel structures, accurate design models need to be established [3].

The lack of knowledge about the force transfer mechanism in adhesive joints and problems involved in the analysis of adhesive joints, such as the singular stress field at sharp corners, 
are just some of the problems contributing to the difficulty associated with developing accurate design models. In addition to the above-mentioned difficulties, the design of adhesively bonded joints in steel members creates additional complexities. Unlike concrete structures, there are, for example, a number of modes such as debonding along the steeladhesive or CFRP-adhesive interfaces, delamination of the CFRP laminate and cohesive failure of the adhesive. In some cases, more complex failure modes - involving a combination of several modes - have also been observed [4]. The failure mode which governs the strength of the bonded joint depends on several parameters. They can be gathered together in the following three main categories.

1. System-specific parameters. These include fiber content and the through-thickness strength of the laminate, adhesive strength, stiffness and ductility, the properties of the primer and compatibility between all the materials in the strengthening system.

2. Parameters related to the method of application, such as surface preparation and pretreatment.

3. Factors related to load effects in the joint, such as the ratio between shear and peeling stress and the degree and location of stress concentration in the joint. These factors are also affected by possible end modifications of the joint, e.g. tapering of the laminate and/or adding adhesive fillets. Furthermore, these end modifications have been shown to affect both the failure load and the failure mode in the strengthened element [5].

When it comes to the above-mentioned parameters, the use of simplified models to make accurate strength predictions for steel-CFRP joints appears to be a fairly difficult task. In general, existing design models can be classified in two main categories; stress-based and fracture mechanics-based models. The strength prediction of adhesive joints in FRPstrengthened steel members using existing stress or energy methods has been the focus of a number of studies. However, in spite of all these efforts, there is still a gap in experimental information in terms of the verification of the applied failure criteria. In other words, based on existing data, no concrete conclusions regarding the accuracy of different design models can be drawn. As a result, making suggestions on the best practice guide is not possible.

The work presented in this paper is mainly concerned with a new design model for adhesively bonded CFRP plates to strengthen steel beams. The new model does not involve the shortcomings often identified in existing design models. The research involves analytical, numerical and experimental work.

\section{The principal of the new method}

In the course of developing the new design model, the following requirements have been considered; the model should (1) be able to predict the strength of the joint with reasonable accuracy, (2) be general and be possible to use irrespective of the characteristics of the system in use and the type of failure mode obtained in that system and (3) be able to treat general loading conditions. The principle of relating the strength of an adhesive joint to the magnitude of a certain stress or strain component at a specific location in the adhesive joint is abandoned in the new proposed model. Instead, it is recognized that, at failure, a unique state of stress (irrespective of which stress component is considered) exists in the adhesive joint. For example, the shear stress distribution at the mid-thickness of the adhesive layer can be regarded as a trait to characterize failure of the joint. 
Mapping the real distribution of shear stresses in the adhesive layer is a very difficult task for the reasons discussed before. However, recognizing that the axial force in the laminate is built up through shear mechanism in the adhesive layer (Figure 1), it should be possible - for a specific strengthening system - to relate the axial force in the laminate at a distance at which the shear lag effects diminish to the load causing failure in that particular strengthening system. Of course, the "uniqueness" of the distribution of shear stresses and the connection to the axial force in the laminate only holds true for adhesive joints with the same material and geometric properties.

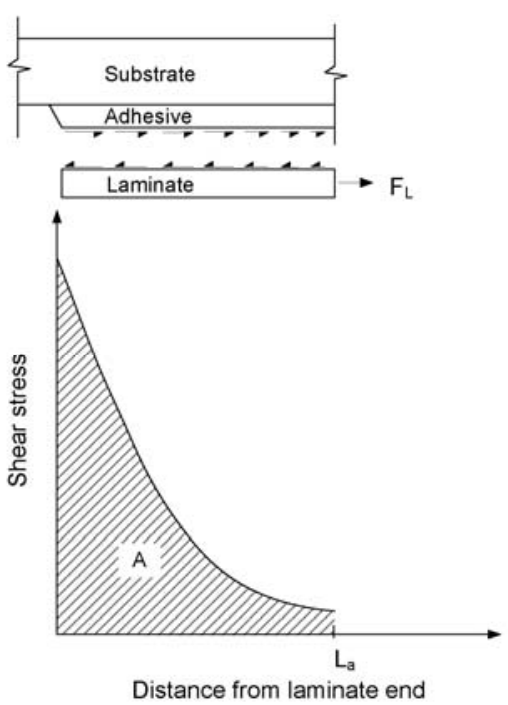

Figure 1. Equivalency of shear stress and force in the laminate at anchorage length

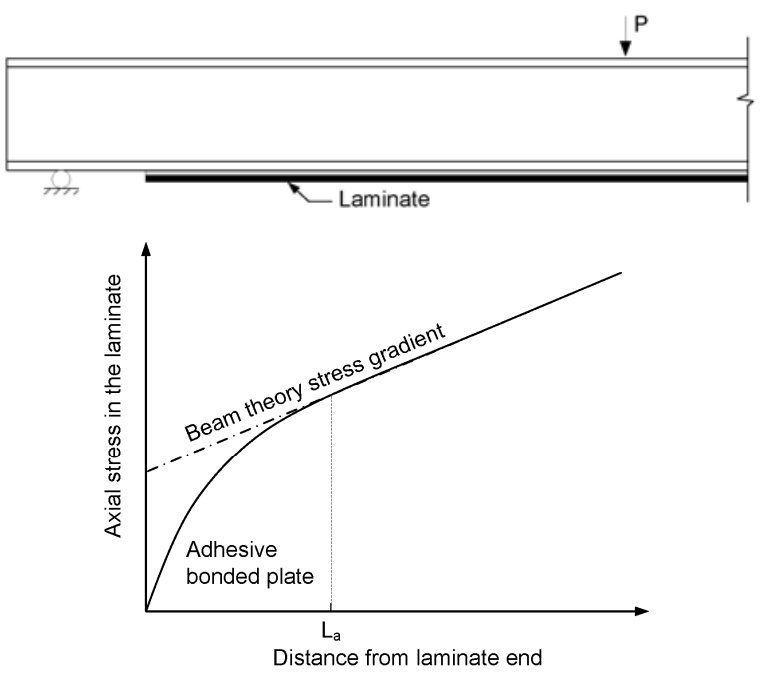

Figure 2. Illustration of the shear lag effect and anchorage length

In other words, it might be possible to consider the axial force linked to the laminate at a certain distance from the laminate end as a global parameter which attains a critical value when failure of the joint takes place. The best location to calculate the axial force in the laminate for this purpose is the point at which the shear lag effect disappears along the bond line, i.e. at the anchorage length. After this location, the axial force in the laminate follows the moment distribution along the beam as calculated by beam theory, see Figure 2. One advantage that follows is that the axial force in the laminate after this location can easily be calculated, based on the assumption of full composite action, without needing to calculate the interfacial stresses in the adhesive layer.

In order to predict the strength of the adhesive joint in a strengthened beam, an ultimate value for the axial force in the laminate at anchorage length is needed. The value of ultimate axial force in the laminate at failure can be obtained experimentally from simple representative specimens that reflect the state of stress in the adhesive joint in the strengthened beam. A comprehensive FE work was carried out to identify the most suitable configuration for a representative test specimen and turned out double-sided strengthened steel plate is the best configuration for this purpose (see Figure 5).

Two major advantages are achieved by this design approach:

- Only one global parameter (the force in the laminate) needs to be determined, which simplifies the design considerably. Local effects, such as stress redistribution due to plasticity and so on, are indirectly taken into account through this global parameter 
- If the representative specimens are well designed, the model can be used for the design of strengthened beams accounting for the actual failure mode of the particular system. For the purpose of designing the joint, the strength of the different constituents does not need to be determined separately.

\section{Formulation of the new design model}

The next step is to relate the critical force in the laminate in the beam to the corresponding value from the representative specimen. Figure 3 shows a schematic of shear stress distributions in the beam and representative specimen, normalized with reference to the maximum shear stress in each specimen. As can be seen, the shear stress distribution in the beam specimen is slightly different from that in the representative specimen. This difference can be attributed to the flexural action in the beam and the consequent curvature in the flange. This effect does not exist in the representative specimen. In other words, in addition to shear lag effect, which is present in both members, the shear distribution in the case of a beam is affected by the flexural behavior of the beam. Assuming that the moment in the beam is taken only by flanges, the strengthened flange of the beam could be simulated by a steel plate subjected to tension, Figure 4. One major difference between the "steel plate" in the representative specimen and the beam is the profile of the axial force, disregarding the bonded laminate. In the former case, the axial force profile is constant along the plate, while, in the latter, the axial force has a gradient which follows the moment diagram over the beam length. This difference implies that the force in the laminate at the anchorage length in the beam would be slightly higher than the corresponding value in the representative specimen. This difference is equivalent to the hatched area in Figure 3. Accordingly, if correspondence is to be obtained between these two members, a correction factor is needed to relate the axial force in the laminate corresponding to failure in the representative specimen to that in the strengthened beam.

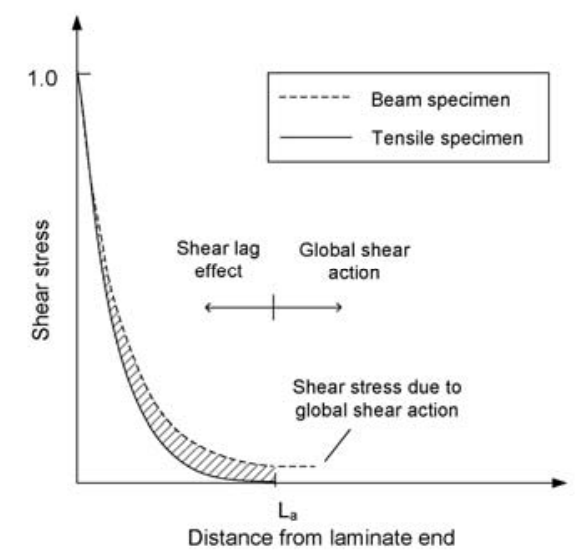

Figure 3. Difference in shear stress distribution in tensile and beam specimens

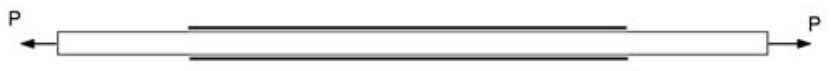

(a)

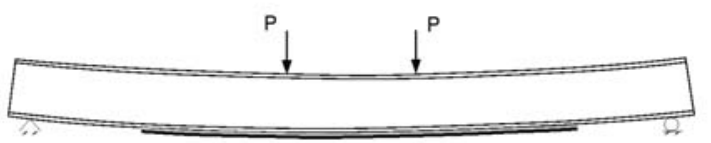

(b)

Figure 4. Difference in deformation of (a) representative specimen and (b) tensile flange of a beam bonded with FRP laminate

This correction factor, $\alpha$, can be easily derived if the distribution of the shear stress in the adhesive layer is known in both members. Distributions can be obtained from any analytical solutions proposed for these configurations. In this study, closed-form solutions developed in [6] and [7] were adopted for double-sided strengthened steel plates and beams strengthened 
with a bonded plate respectively. The correction factor, $\alpha$, could therefore be defined as the ratio of the area under the curve for the beam to that of the representative specimen. The new failure criterion can therefore be formulated as

$$
F_{L B} \leq \alpha F_{L P}
$$

in which $F_{L B}$ is the force in the laminate at the anchorage length in the beam and $F_{L P}$ is the force in the laminate at the anchorage length in the representative specimen, which can be calculated from

$$
F_{L P}=P_{u} \frac{E_{l} A_{l}}{2\left(E_{l} A_{l}\right)+E_{s} A_{s}}
$$

Where $P_{u}$ is the ultimate load of the representative specimen, $F_{L B}$ can easily be calculated from beam theory and transformed section analysis for the design loading condition assuming full composite action.

$\alpha=\frac{F_{2}}{F_{1}}$ where $F_{2}=\frac{\Delta \tau b_{1}}{\beta}\left(1+\sinh \left(\beta L_{a}\right)-\cosh \left(\beta L_{a}\right)\right)+n \gamma\left(M\left(L_{a}\right)-M(0)\right)$ and $F_{1}=\frac{\psi b_{a}}{-\lambda}\left(e^{-\lambda L_{a}}-1\right)$. In these equations $\Delta \tau=\psi-\tau_{\text {Global.shear }}$ in which $\tau_{\text {Global.shear }}=\frac{V(0) \gamma n}{b_{a}}$ and $\beta=\sqrt{\frac{K_{s}}{E_{l} b_{l} t_{l}}}$ in which $K_{s}=\frac{G_{a} b_{l}}{t_{a}}$ and $\gamma=\frac{Q}{I_{s t r}}$.

$\psi$ : The maximum shear stress expected in the adhesive layer at the end of the laminate in the beam and is expressed as $\psi=\frac{\lambda \eta \varepsilon}{b_{a} f_{2}}$ in which $\varepsilon=\frac{M(0)}{E_{b} W_{b}}$ and $\eta=0.188 \frac{E_{l}}{E_{b}}+0.97$. The anchorage length is $L_{a}=\frac{4.6}{\lambda}$ where $\lambda=\sqrt{\frac{f_{2}}{f_{1}}}$ in which $f_{1}=\frac{t_{a}}{G_{a} b_{a}}$ and $f_{2}=\frac{2}{E_{s} A_{s}}+\frac{1}{E_{l} A_{1}}$. In these equations, $A_{1}, E_{l}$ : cross-sectional area and modulus of elasticity of the laminate, $A_{s}$, $E_{s}$ : cross-sectional area and modulus of elasticity of the steel plate in representative specimen, $t_{a}$ : thickness of the adhesive layer, $b_{a}$ : width of the laminate, $G_{a}$ : shear modulus of the adhesive, $E_{b}$ : modulus of elasticity of steel in the beam, $W_{b}$ : sectional modulus of unstrengthened beam, $M(x), V(x)$ : bending moment and shear force distribution in the beam in which $x$ starts at the laminate end, $n$ : Stiffness ratio defined as the modulus of elasticity of the laminate to the modulus of elasticity of the beam material, $Q$ : first moment of inertia of the laminate with respect to neutral axis of strengthened section and $I_{s t r}$ : second moment of inertia of strengthened section. 


\section{Experimental work}

Two series of tests were conducted in the experimental part of this study. In the first series, double-sided strengthened steel plates, referred to as representative specimens, were chosen as representative test specimens. The second series consisted of 6 full-scale steel beams, strengthened on the tension flange with adhesively bonded CFRP laminates. A schematic of the representative specimens is shown in Figure 5. Three series of specimens were manufactured using two strengthening systems produced by Sika ${ }^{\circledR}$ and STO ${ }^{\circledR}$. The first series, referred to as the "STO series", consisted of the STO system (StoFRP plate M50C, StoBPE primer 50 super and StoBPE lim 567 epoxy). The second series was made from the Sika system (Sika carbodur S512 and Sikadur 330 epoxy) and the third was manufactured from Sika but with a different laminate type (Sika carbodur H514 and Sikadur 330 epoxy) referred to as "Sika 512" and "Sika 514" respectively. See Table 1 for the materials used in each series. In order to prevent yielding in representative specimens, high strength steel (S690) was used. The material properties are presented in Table 2. The thickness of the adhesive layer was measured in all specimens after curing the adhesive. The thickness was measured as 2 $\mathrm{mm}$ in the STO and the Sika 514 series, while it was $2.5 \mathrm{~mm}$ in the Sika 512 series. The tolerance of the thickness was $\pm 0.1 \mathrm{~mm}$.
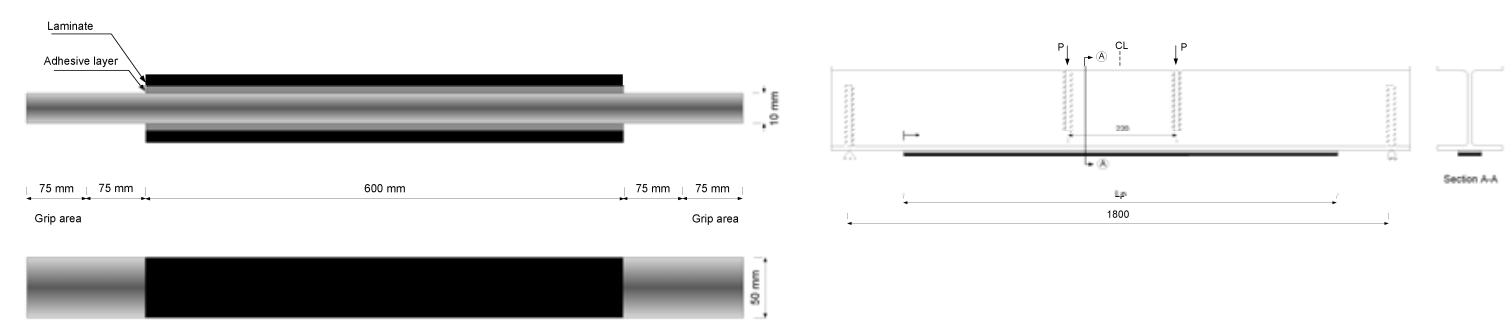

Figure 5. Nominal dimensions of representative specimens

Figure 6. Configuration of beam specimens

Table 1. Materials used in manufacturing test specimens

\begin{tabular}{lccl}
\hline Series & \multicolumn{1}{c}{ Laminate } & Primer & \multicolumn{1}{c}{ Adhesive } \\
\hline STO & StoFRP plate M50C & StoBPE primer 50 super & StoBPE lim 567 epoxy \\
Sika 512 & Sika carbodur S512 & - & Sikadur 330 epoxy \\
Sika 514 & Sika carbodur H514 & - & Sikadur 330 epoxy \\
\hline
\end{tabular}

Table 2. Material properties used to manufacture specimens

\begin{tabular}{lcccccc}
\hline Material & $\begin{array}{c}\text { E-modulus } \\
{[\mathrm{GPa}]}\end{array}$ & $\begin{array}{c}\text { Poisson's } \\
\text { ratio }\end{array}$ & $\begin{array}{c}\text { Thickness } \\
{[\mathrm{mm}]}\end{array}$ & $\begin{array}{c}\text { Width } \\
{[\mathrm{mm}]}\end{array}$ & $\begin{array}{c}\text { Tensile } \\
\text { strength } \\
{[\mathrm{MPa}]}\end{array}$ & $\begin{array}{c}\text { Strain at } \\
\text { failure }^{\mathrm{b}} \\
{[\%]}\end{array}$ \\
\hline Sikadur ${ }^{\circledR}$ 330 & $4.5^{\mathrm{a}}$ & 0.3 & - & - & 32 & 0.65 \\
Sto BPE Lim 567 & $7^{\mathrm{a}}$ & 0.3 & - & - & 26 & 0.82 \\
Sika $^{\circledR} \quad$ carbodur $^{\circledR}$ & $165^{\mathrm{b}}(200)$ & 0.3 & 1.2 & 50 & $3100^{\mathrm{b}}$ & $>1.7$ \\
Sika $^{\circledR} \quad$ carbodur $^{\circledR}$ & $300^{\mathrm{b}}(325)$ & 0.3 & 1.4 & 50 & $1500^{\mathrm{b}}$ & $>0.45$ \\
StoFRP plate M50 C & $260^{\mathrm{b}}(300)$ & 0.3 & 1.4 & 50 & 1100 & 0.8 \\
Steel (S690) & 200 & 0.29 & 10 & 50 & - & - \\
\hline
\end{tabular}

${ }^{\mathrm{a}}$ The E-modulus was measured after 7 days. ${ }^{\mathrm{b}}$ Information provided by the manufacturer. Values in brackets were calculated from the representative specimens in this study.

Three series of beam specimens were manufactured using the strengthening systems mentioned in Table 1. The specimens were manufactured using HEA 180 steel section with 
quality of S355 and modulus of elasticity of $200 \mathrm{GPa}$. $10 \mathrm{~mm}$ thick rectangular stiffeners were welded at supports and under the point loads in order to prevent buckling in the web. The configuration of beam specimens is presented in Figure 6. The same width of the laminate and thickness of the adhesive layer as in representative specimens in each series were maintained in beam specimens. Different laminate lengths were chosen in bream specimens as shown in the first column of Table 3.

The representative specimens were tested using an MTS universal testing machine with a capacity of $1000 \mathrm{kN}$ in the displacement control mode at a rate of $0.5 \mathrm{~mm} / \mathrm{min}$. The beam specimens were tested in a four-point bending configuration using the rig shown in Figure 7. In order to ensure the same strain transfer rate in the laminates as in the representative specimens, the beam was loaded under displacement control at a rate of $1 \mathrm{~mm} / \mathrm{min}$ until failure.

\section{Results}

Table 3 presents the predicted loads for different specimens tested in this study. These values are plotted in Figure 8. A good correlation between the predicted and measured values can be seen in this figure. The accurate predictions based on the proposed design model indicate that the idea of considering the axial force in the laminate as an indicator of failure in joints is practical.

Table 3. Strength prediction based on proposed model

\begin{tabular}{l|ccccccc}
\hline Specimen & $\begin{array}{c}L_{P} \\
{[\mathrm{~mm}]}\end{array}$ & $\begin{array}{c}L_{a} \\
{[\mathrm{~mm}]}\end{array}$ & $\begin{array}{c}F_{L B} \\
{[\mathrm{kN}]^{\mathrm{a}}}\end{array}$ & $\begin{array}{c}F_{L P} \\
{[\mathrm{kN}]^{\mathrm{b}}}\end{array}$ & $\alpha$ & $\begin{array}{c}\text { Predicted load } \\
{[\mathrm{kN}]}\end{array}$ & $\begin{array}{c}\text { Measured } \\
{[\mathrm{kN}]}\end{array}$ \\
\hline STO-500 & 500 & 68 & 13.25 & 23.36 & 1.278 & 225 & 241 \\
STO-800 & 800 & 68 & 9.47 & 23.36 & 1.307 & 322 & 326 \\
Sika512-600 & 600 & 76 & 6.62 & 13.54 & 1.209 & 247 & 243 \\
Sika514-500 & 500 & 87 & 14.67 & 14.68 & 1.324 & 132 & 148 \\
Sika514-700 & 700 & 87 & 12.44 & 14.68 & 1.346 & 159 & 170 \\
Sika514-1000 & 1000 & 87 & 9.67 & 14.68 & 1.401 & 212 & 208 \\
& & & & & & & \\
\hline
\end{tabular}

\footnotetext{
${ }^{\mathrm{a}}$ The axial force in the laminate in beam specimens for a reference load of $100 \mathrm{kN}$

${ }^{b}$ The values correspond to the mean minus one standard deviation of the results obtained from representative specimens.
}

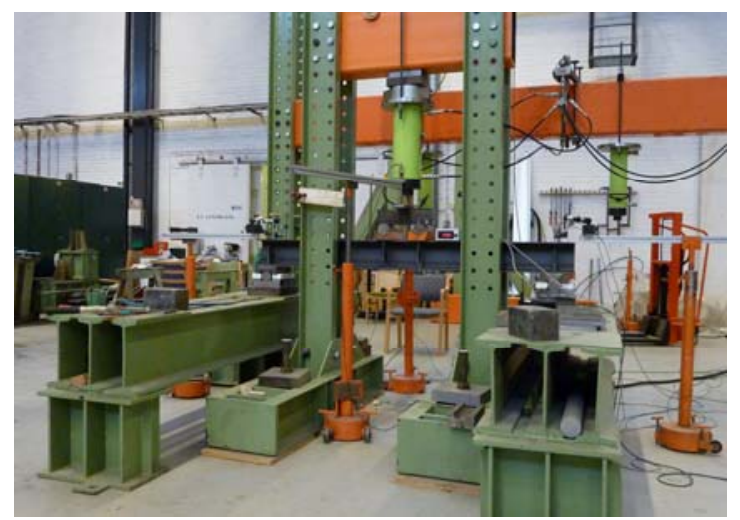

Figure 7. Test set-up for beam specimens

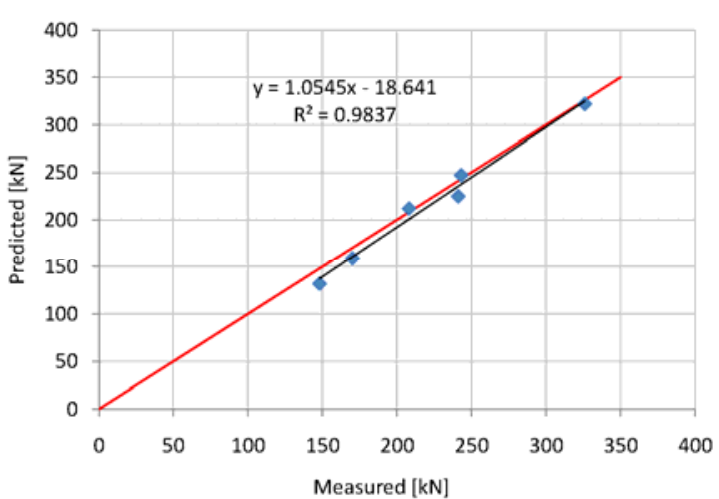

Figure 8. Measured and predicted strength of beam specimens 
The failure mode observed in all specimens was debonding at the steel-adhesive interface, see Figure 9.

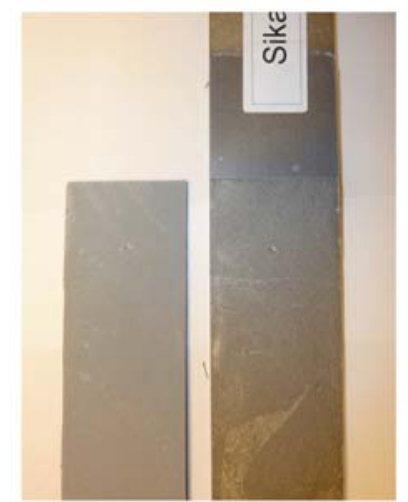

(a)

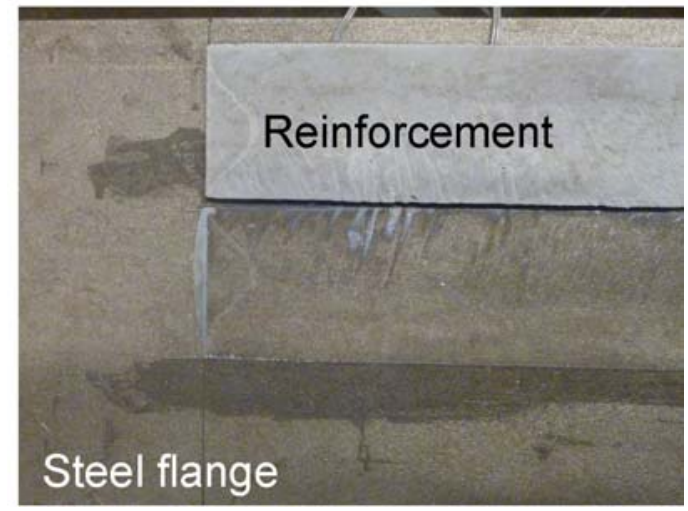

(b)

Figure 9. Failure mode in: (a) representative and (b) beam specimens

\section{Conclusions}

The proposed design model was found to be accurate in terms of predicting the ultimate load of steel beams strengthened with bonded CFRP laminates. The model eliminates the laborious and lengthy analytical calculations.

\section{References}

[1] Hollaway, L. C., Teng, J. G., "Strengthening and rehabilitation of civil infrastructures using fibre-reinforced polymer (FRP) composites", Woodhead Publishing Limited, Cambridge, UK, 2008.

[2] Linghoff, D., “Steel members strengthened with carbon fibre reinforced polymers”, $\mathrm{PhD}$ thesis, Chalmers University of Technology, Sweden, 2009.

[3] Buyukozturk, O., Gunes, O., Karaca, E., "Progress on understanding debonding problems in reinforced concrete and steel members strengthened using FRP composites", Construction and Building Materials, V.18, 2004, 9-19.

[4] Al-Emrani, M., Kliger, R., "Experimental and numerical investigation of the behavior and strength of composite steel-CFRP members”, Advances in Structural Engineering, V.9, No.6, 2006, 29-41.

[5] Haghani, R., "Analysis of geometrically modified adhesive joints in steel beams strengthened with composite laminates", Licentiate thesis, Chalmers University of Technology, Sweden, 2008.

[6] Bocciarelli, M., Colombi, P., Fava, G., Poggi, C., "Prediction of debonding strength of tensile steel/CFRP joints using fracture mechanics and stress based criteria", Engineering Fracture Mechanics, 2009, V. 76, 299-313.

[7] Roberts, T. M., "Approximate analysis of shear and normal stress concentrations in the adhesive layer of plated RC beams”, The Structural Engineer, V. 67, No. 12, 1989, 229-233. 